\title{
Differential Effects of Nicotine and Complex Housing on Subsequent Experience-Dependent Structural Plasticity in the Nucleus Accumbens
}

\author{
Derek A. Hamilton and Bryan Kolb \\ University of Lethbridge
}

\begin{abstract}
Drugs and other forms of experience (e.g., complex housing) share the ability to alter the dendritic fields of cortical and subcortical neurons. Although such modifications are typically considered advantageous, recent research has demonstrated that psychomotor stimulants (cocaine and amphetamine) block subsequent experience-dependent structural plasticity in the nucleus accumbens (NAcc) and parietal neocortex. The authors investigated whether these findings generalize to another commonly used stimulant (nicotine) and further asked whether prior experience blocks subsequent nicotine-related structural plasticity. Rats were given daily injections of nicotine (or saline) for 14 days either before (Experiment 1) or after (Experiment 2) 2.5-3.0 months of complex (or standard) housing. Nicotine blocked housing-related increases in dendritic branching, length, spine density, and total spines in NAcc; however, complex housing did not block the effects of nicotine. The findings indicate that there are important differences in the capacity of drugs and experience to influence subsequent modifications in dendritic structure.
\end{abstract}

Keywords: stimulants, enrichment, Golgi, camera lucida, mesolimbic dopamine system

Beginning with the seminal work of Rosenzweig and colleagues (e.g., Rosenzweig \& Bennett, 1978; Rosenzweig, Krech, Bennett, $\&$ Diamond, 1962), the notion that experience modifies the brain has been supported by an impressive body of empirical data and has since become a core theoretical concept in the neurosciences (Greenough \& Bailey, 1988; Kolb \& Whishaw, 1998). Experiencedependent changes in neurochemistry, anatomy, electrophysiology, and neuronal structure have been demonstrated following many forms of experience including sensory stimulation, chronic drug administration, and specific training in behavioral and cognitive tasks (Greenough, Withers, \& Wallace, 1990; Kolb, Forgie, Gibb, Gorny, \& Rowntree, 1998; Kolb \& Whishaw, 1998; van Pragg, Kempermann, \& Gage, 2000). Several studies have demonstrated that complex housing, drugs, and specific training can increase the length of dendrites and spine density, the primary sites of excitatory synapses (Harris \& Kater, 1994; Nimchinsky, Sabatini, \& Svoboda, 2002), on individual neurons, effectively increasing the amount of space available for synaptic connections. Such changes are thought to be one of the primary mechanisms by which the brain adapts to environmental demands and, therefore,

Derek A. Hamilton and Bryan Kolb, Department of Psychology and Neuroscience, Canadian Centre for Behavioural Neuroscience, University of Lethbridge, Lethbridge, Alberta, Canada.

This research was supported by an Alberta Heritage Foundation for Medical Research postdoctoral fellowship to Derek A. Hamilton and by National Science and Engineering Research Council of Canada Grant 10264 to Bryan Kolb. We thank Grazyna Gorny for teaching the camera lucida technique to Derek A. Hamilton, and we thank Gergely Silasi for assistance with tissue preparation and staining. We also thank Elise McHugh for assistance in preparing this article.

Correspondence concerning this article should be addressed to Derek A. Hamilton, who is now at the Department of Psychology, University of New Mexico, Logan Hall, Room 166, MSC03 2220, Albuquerque, NM 87131. E-mail: dahamilt@unm.edu may represent an important component of the neural substrate of learning and memory.

Although neural plasticity is generally considered advantageous, several studies have revealed that modifications in the structure of dendrites and dendritic spines are associated with certain pathological conditions (Fiala, Spacek, \& Harris, 2002; Purpura, 1974), and may underlie maladaptive behaviors such as those involved in drug addiction. For example, psychostimulant drugs, such as amphetamine, nicotine, and cocaine, lead to increases in dendritic arborization and spine density in the nucleus accumbens (NAcc) and prefrontal neocortex (Brown \& Kolb, 2001; Kolb, Gorny, Li, Samaha, \& Robinson, 2003; Li, Kolb, \& Robinson, 2003; Robinson \& Kolb, 1997, 1999), brain regions thought to be involved in stimulant addiction (Pich et al., 1997) and craving (Miyata \& Yanagita, 2001). These drug-induced modifications in dendritic structure may offer some insight into the puzzling pattern of cognitive impairments associated with the cessation of psychostimulant use in addicts. Whereas the acute psychoactive effects of nicotine are typically associated with cognitive enhancement (Bernert, Sustrova, Sovcikova, Seidl, \& Lubec, 2001; Jarvik, 1991; Le Houezec et al., 1994; Rezvani \& Levin, 2001), long-term stimulant abuse and withdrawal are associated with a diverse array of cognitive impairments (reviewed in Rogers \& Robbins, 2001). Although it is tempting to link these impairments to nicotinic receptor upregulation associated with withdrawal, receptor upregulation in the rat appears to be transient, lasting as little as 1 week (Collins, Romm, \& Wehner, 1990) and up to 1 month (Trauth, Seidler, McCook, \& Slotkin, 1999). In contrast, the behavioral consequences following chronic stimulant exposure last much longer. For example, behavioral sensitization to amphetamine in rats has been shown to persist for at least 1 year following cessation (Paulson, Camp, \& Robinson, 1991).

The shared ability of stimulants and complex housing to alter dendritic structure and spine density prompted Kolb, Gorny, Li, et 
al. (2003) to ask whether drugs and experience might interact with respect to their influence on structural plasticity. Because the capacity for structural plasticity is likely to be limited, they hypothesized that prior exposure to stimulants may diminish the ability of later experience to elicit structural changes in dendrites that are thought to be involved in normal behavioral adaptation. To test this hypothesis, they gave rats repeated injections of cocaine or amphetamine and subsequently housed them in a complex environment for 3 months. Prior exposure to amphetamine or cocaine limited subsequent housing-related structural plasticity in the NAcc shell, a subcortical region associated with incentive motivation and reward (Berridge \& Robinson, 2003; Kelley, 1999), and to a lesser extent in the parietal neocortex (Par1). To further investigate these effects, we undertook the present study to replicate the study of Kolb, Gorny, Li, et al. with another commonly used psychostimulant drug (i.e., nicotine) and to extend their findings by investigating whether complex housing blocks subsequent structural plasticity due to nicotine exposure. If the effects of nicotine on subsequent experience-dependent structural plasticity are similar to other psychostimulants, we expect that subsequent experience-dependent increases in dendritic arbor and spines will be limited. Further, if the effects of prior experience-dependent structural plasticity have generality, then we expect that complex housing will also diminish or block subsequent nicotine-related structural plasticity.

\section{General Method}

\section{Subjects}

A total of 48 female Long-Evans (hooded) rats bred at the University of Lethbridge vivarium (originally from Charles River stock, St. Constant, Quebec) were used in this study. All rats weighed between 200-250 g at the beginning of the study. Prior to experimentation, all rats were housed in groups of 3 in plastic hanging cages. The animal colony rooms were maintained on a $12-12 \mathrm{hr}$ light-dark cycle with the light cycle beginning at 0700 . The specific housing methods used in this study are described in further detail below. All experimental procedures and housing conditions were approved by the University of Lethbridge Institutional Animal Care and Use Committee.

\section{Nicotine Administration}

For 14 consecutive days, rats were given subcutaneous nicotine (or saline) injections $(0.3 \mathrm{mg} / \mathrm{kg})$ in the nape of the neck. This dose of nicotine is estimated to be roughly comparable to a human smoking four cigarettes per day (Levin, Wilkerson, Jones, Christopher, \& Briggs, 1996; see also Fung \& Lau, 1989, and Murrin, Ferrer, Zeng, \& Haley, 1987). All injections were performed in a small room near the animal colony facilities and were given approximately $2-3 \mathrm{hr}$ after the onset of the light phase. Prior to being returned to the animal colony (or complex cages), rats were left in the injection room for $30 \mathrm{~min}$ following nicotine or saline administration.

\section{Behavior}

One day prior to injections and following injections on Days 7 and 14, rats were individually placed into a Digiscan activity monitor $(40 \mathrm{~cm} \times 40$ $\mathrm{cm} \times 30 \mathrm{~cm}$; Omnitech, Columbus, $\mathrm{OH}$ ) containing a grid of horizontal infrared beams for $10 \mathrm{~min}$. A count was incremented each time the rat's body disrupted a beam, providing an index of locomotor behavior. The total number of beam crosses during the 10-min session was analyzed using a repeated measures analysis of variance (ANOVA) with day as a single within-subjects factor. Drug (both experiments) and housing condition (Experiment 2 only) were included as between-subjects factors.

\section{Housing}

Two housing conditions were used. In the standard housing condition, rats were housed in plastic hanging cages in groups of 3 . Fresh bedding and clean cages were provided twice per week. In the complex housing condition, rats were housed in large steel cages $(2 \mathrm{ft} \times 4 \mathrm{ft} \times 6 \mathrm{ft})$ in groups of 6 . Once per week, the cages were cleaned, the bedding was replaced, and a number of novel objects were spread around the bottom of the cage. Near the end of each light cycle, various types of pasta were spread around the cages to encourage exploration, and food rewards (e.g., pretzels) were placed at the top of the mesh cage to encourage climbing. Rat chow and water were available ad libitum in both housing conditions. An equal number of nicotine-treated and saline-treated rats were housed in each complex environment (i.e., 3 per cage). Standard cages consisted of 2 rats from one drug treatment condition and 1 rat from the other.

\section{Golgi-Cox Staining and Analyses}

Rats were deeply anesthetized with an overdose of sodium pentobarbital and perfused intracardially with $0.9 \%$ (wt/vol) saline, resulting in exsanguination. The brains were extracted and immersed in Golgi-Cox solution (Glaser \& Van der Loos, 1981) for 14 days and subsequently immersed in $30 \%$ (wt/vol) sucrose for at least 3 days. The brains were then cut into coronal sections (200 $\mu \mathrm{m}$ thick) on a vibrating microtome, mounted on slides, and stained according to the procedures described by Gibb and Kolb (1998).

Layer III pyramidal neurons in Par1 (Zilles's area Par1; Zilles, 1985) and medium spiny neurons in the shell of the NAcc (Zilles's area NAcc; see Figure 1) were selected for analysis because they have previously been shown to be sensitive to either psychostimulant drugs or housing conditions. Both nicotine and complex housing have been shown to increase dendritic length and spine density in NAcc (Brown \& Kolb, 2001; Kolb, Gorny, Li, et al., 2003). In contrast, complex housing (Kolb, Gorny, Li, et al., 2003), but not nicotine (Brown \& Kolb, 2001), modifies dendritic structure in Par1. The brain regions of interest were first identified at low power (100× magnification), and five neurons from each hemisphere were traced in each region at a higher power $(250 \times$ final magnification) using the camera lucida technique (see Figure 1 for a representative example of a stained NAcc neuron and terminal segment showing the staining of dendritic spines). Selection was limited to neurons which were not obscured by stain precipitate, blood vessels, astrocytes, or other artifacts, and had intact dendritic fields that were well impregnated and visible within a single section. Dendritic branching was measured by counting bifurcations on each dendrite (Coleman \& Riesen, 1968); dendritic segments prior to the first bifurcation from the cell body were designated a first-order branch, second-order branch following the first-bifurcation, and so on. Dendritic length was measured using a Sholl analysis of ring intersections (Sholl, 1981). A series of concentric rings at $20 \mu \mathrm{m}$ intervals (calibrated to the final magnification of $250 \times$ ) was transferred to a transparency which was centered over the cell body. The total number of intersections between rings and each dendritic branch and rings was counted. Spine density was measured by tracing the length of a dendritic terminal tip ( $>20 \mu \mathrm{m}$ long) at high power $(2000 \times$ final magnification) using the camera lucida procedure, computing the length of the dendritic segment, and counting the number of spines along the segment. Spines were counted on third-order (or greater) terminal segments for NAcc neurons and Par1 apical dendrites, and on fourth-order (or greater) terminal segments for Par1 basilar dendrites. Spine density was quantified on five terminal segments for each hemisphere and separately for both apical and basilar dendritic fields on Par1 neurons. An estimate of total spines per neuron was determined by calculating the product of the dendritic length and spine density. Hemi- 

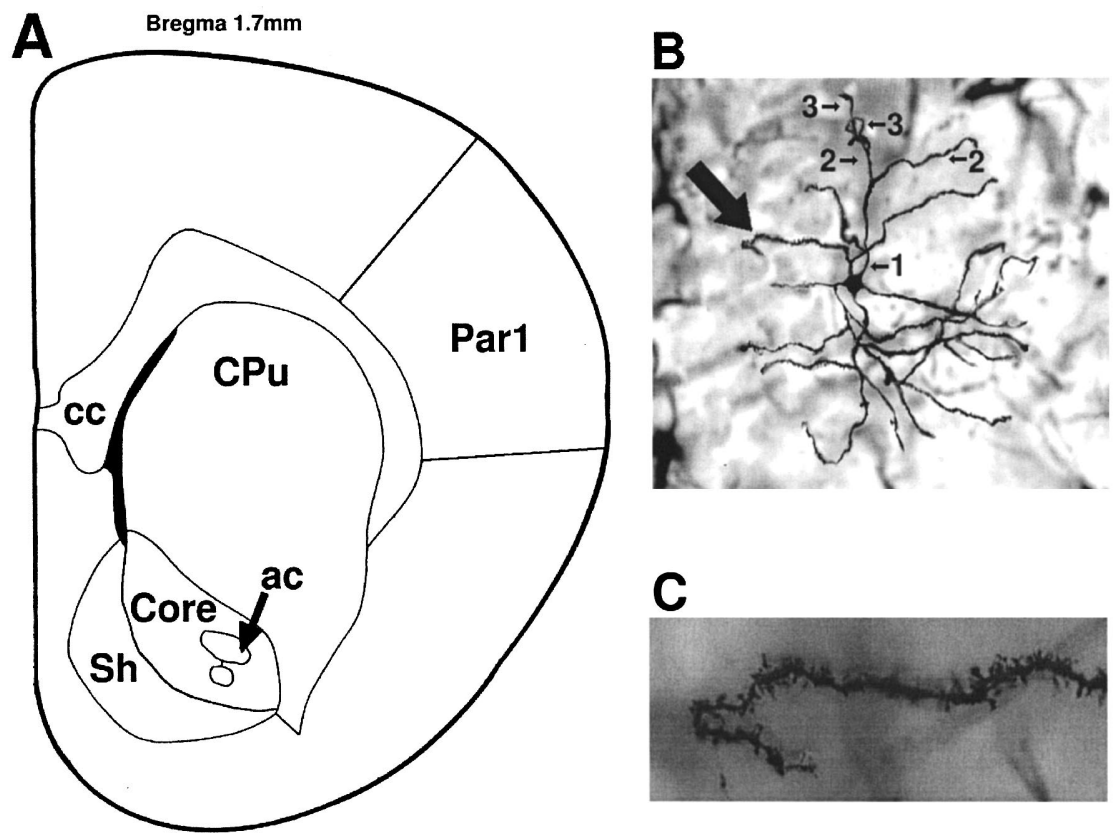

Figure 1. A: Section showing the location of the nucleus accumbens (Zilles's NAcc) core and shell (Sh). Also shown are the parietal neocortex (Par1), corpus callosum (cc), anterior commissure (ac), and caudate putamen $(\mathrm{CPu})$. Adapted from Figure 9 of The Cerebral Cortex of the Rat (p. 17), by K. Zilles, 1985, Berlin, Germany: Springer-Verlag. Copyright 1985 by Springer-Verlag. Adapted with permission. B: A representative medium spiny neuron from the NAcc shell showing the quality of the staining. Small arrows point to branch segments, and the corresponding numbers indicate branch order $(1=1$ st order and so on). The large arrow points to a terminal segment shown in Figure 1C at higher magnification. C: A terminal segment showing staining of dendritic spines. Note that portions of the terminal segment are not in the focal plane of the microscope; therefore, some of the dendritic spines appear blurred.

sphere was included as a factor and used as the unit of analysis for all statistical analyses of dendritic length, branch order, spine density, and total spines. All dendritic measurements were analyzed in separate threefactor (housing, drug, and hemisphere) ANOVAs and followed by post hoc comparisons using Fisher's least significant difference. Separate ANOVAs were conducted for apical and basilar dendritic fields on Par1 neurons.

\section{Experiment 1}

\section{Method}

A total of 24 rats were used in Experiment 1. Beginning on Postnatal Day 90 , rats were given daily injections of nicotine $(n=12)$ or saline $(n=$ 12) for 14 days. Open-field activity was measured 1 day prior to the first injection and following injections on Days 7 and 14. One day following the final injection, half of the rats from each drug group were housed in either standard or complex cages for 90 days. The brains were extracted and processed for Golgi-Cox staining and analysis.

\section{Results}

All statistical analyses were performed using SPSS 11.5 for Windows (SPSS, Chicago, IL), and an alpha level of $p<.05$ was adopted for all statistical tests.

\section{Behavior}

Mean beam crosses for nicotine-treated and saline-treated rats for the 3 days of activity monitoring are shown in Figure 2. Neither the drug or day main effects reached statistical significance (both $p \mathrm{~s}>.09)$, however, there was a significant interaction, $F(2,44)=$ $7.67, p=.001$. The interaction was attributable to a significant effect of drug on the final injection day, nicotine $>$ saline, $F(1$, $22)=6.70, p=.017$, which was not present prior to injections or on Injection Day 7 (both $p$ s $>$.29).

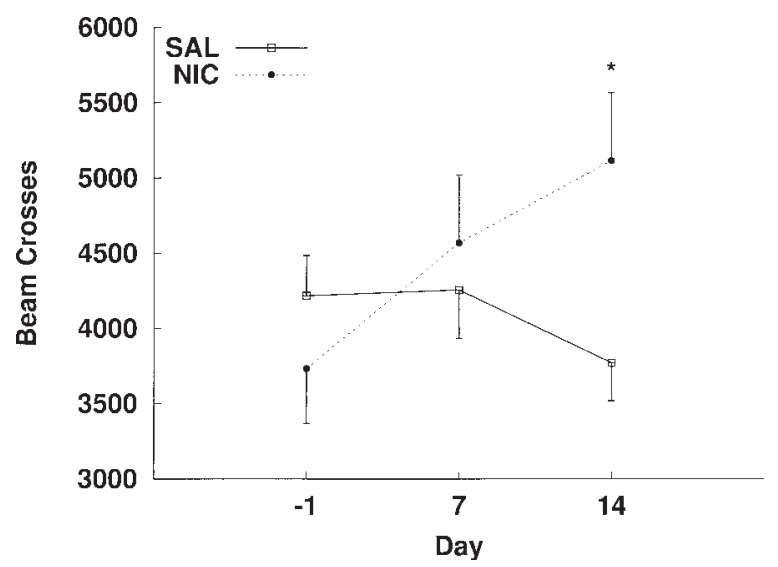

Figure 2. Mean ( \pm 1 SEM) number of beam crosses during $10 \mathrm{~min}$ in an open-field activity monitor for saline-treated (SAL; $n=12)$ and nicotinetreated (NIC; $n=12$ ) rats 1 day prior to injections (Day -1 ) and on Days 7 and 14 of injections. $* p<.05$. 


\section{Brain Weight}

Mean brain weights (measured at the time of perfusion) for each group from each experiment are shown in Table 1. Brain weights were analyzed using a two-factor (Housing $\times$ Drug) ANOVA. In the present experiment, brain weight was lowest in the salinetreated, standard-housed rats and highest in nicotine-treated, complex-housed rats; however, there were no main effects of drug or housing on postmortem brain weight (both $p \mathrm{~s}>.45$ ), nor was there a significant interaction $(p>.76)$.

\section{Golgi-Cox Analysis: NAcc}

Six of the brains contained staining that was inadequate for quantification of spines and were, therefore, excluded from the following analyses. The number of brains ultimately represented per group were as follows: saline treated, standard housed $(n=5)$, saline treated, complex housed $(n=4)$, nicotine treated, standard housed $(n=5)$, nicotine treated, complex housed $(n=4)$. We planned to conduct comparisons of groups from both experiments reported here; therefore, all dendritic measures were computed as the percentage of the mean for the baseline (saline-treated, standard-housed) condition to control for any differences in the quality of the staining across experiments. ${ }^{1}$ Because no interactions involving hemisphere or hemisphere main effects were significant, group means were averaged across hemisphere for Figures 3 and 4.

Dendritic length. As shown in Figure 3A, both nicotine and complex housing were associated with increases in dendritic length on medium spiny neurons. The main effect of housing condition was not significant, $F(1,28)=2.11, p=.157$, and the drug main effect only approached significance, nicotine $>$ saline, $F(1,28)=3.87, p=.059$, however, there was a significant Drug $\times$ Housing interaction, $F(1,28)=4.67, p=.039$. The interaction was attributable to a housing effect in saline-treated rats (complex $>$ standard, $p=.004$ ) that was not observed in nicotinetreated rats $(p=.86)$. Post hoc analyses confirmed that dendritic length was greater in each of the three treatment conditions (nicotine, complex housing, or both) compared with the baseline (saline-treated, standard-housed) condition (all $p \mathrm{~s}<.021$ ). There were no significant differences among treatment groups (all $p \mathrm{~s}>$ .61), further suggesting that complex housing did not have an additional effect on dendritic length after nicotine exposure. None of the interactions with hemisphere or the hemisphere main effect were significant (all $p \mathrm{~s}>.20$ ).

Dendritic branches. Mean dendritic branches for each group are shown in Figure 3B. None of the interactions involving hemisphere or the hemisphere main effect were significant (all $p \mathrm{~s}>$

Table 1

Mean (SEM) Brain Weights in Grams for Each Group in Experiments 1 and 2

\begin{tabular}{cccccc}
\hline & \multicolumn{2}{c}{ Saline $(n=6)$} & & \multicolumn{2}{c}{ Nicotine $(n=6)$} \\
\cline { 2 - 3 } \cline { 5 - 6 } Experiment & Standard & Complex & & Standard & Complex \\
\hline 1 & $2.08(0.036)$ & $2.12(0.042)$ & & $2.12(0.041)$ & $2.14(0.030)$ \\
2 & $1.96(0.027)$ & $2.02(0.025)$ & & $1.97(0.043)$ & $2.07(0.032)$ \\
\hline
\end{tabular}

.33). The main effect of housing was significant, complex $>$ standard, $F(1,28)=4.63, p=.04$, however, neither the drug main effect, $F(1,28)=1.29, p=.27$, nor the Housing $\times$ Drug interaction, $F(1,28)=2.36, p=.14$, were significant. Post hoc group comparisons revealed that total dendritic branch estimates were greater in each of the three treatment conditions compared with the baseline condition (all $p \mathrm{~s}<.046$ ). There were no significant differences among treatment groups (all $p$ s $>.46$ ). Additional post hoc analyses revealed that both nicotine-treated groups had significantly more higher order (sixth or higher) branches than their respective saline-treated condition (data not shown; both $p$ s $<.022)$.

Spine density. Mean spine density values for each group are shown in Figure 3C. The main effect of housing was not significant, $F(1,28)=2.11, p=.157$. There was, however, a significant main effect of drug, nicotine $>$ saline, $F(1,28)=8.39, p=.007$, as well as a significant Housing $\times$ Drug interaction, $F(1,28)=$ $6.68, p=.015$. This interaction was attributable to a significant housing effect in saline-treated rats (complex $>$ standard, $p<$ $.001)$ that was not observed in nicotine-treated rats $(p=.84)$. Post hoc analyses revealed that spine density was greater in each of the three treatment conditions compared with the baseline condition (all $p$ s $<.009$ ). There were no significant differences among treatment groups (all $p \mathrm{~s}>.33$ ). None of the interactions involving hemisphere or the hemisphere main effect were significant (all ps $>$.16).

Total spines. Mean total spine values for each group from Experiment 1 are shown in the left side of Figure 4. The main effect of housing failed to reach statistical significance, $F(1,28)=$ $3.04, p=.09$. There was, however, a significant main effect of drug, nicotine $>$ saline, $F(1,28)=8.18, p=.008$, as well as a significant Housing $\times$ Drug interaction, $F(1,28)=8.19, p=.008$. The interaction was attributable to a significant housing effect in saline-treated rats (complex $>$ standard, $p=.003$ ) that was not observed in nicotine-treated rats $(p=.43)$. Post hoc group comparisons revealed that there were significantly more spines in each of the three treatment conditions compared with the baseline condition (all $p \mathrm{~s}<.003$ ), and there were no significant differences among treatment groups (all $p \mathrm{~s}>.43$ ). None of the interactions involving hemisphere or the hemisphere main effect were significant (all $p s>.16)$.

\footnotetext{
${ }^{1}$ Experiments 1 and 2 were conducted several months apart with staining conducted by different individuals. Because the treatment was identical for the saline-treated, standard-housed rats, this correction adjusts for any differences in the quality of staining. We estimated that the difference in dendritic measurements for the baseline condition in the two experiments due to differences in staining quality was approximately $2.4 \%$. Note that the effects of complex housing or nicotine alone using the corrected measures were similar across experiments, and that this correction is only a linear transformation of the original data. It, therefore, does not affect the outcome of the statistical analyses within each experiment. Presenting effects as percentage of the baseline condition also facilitates comparisons between studies conducted in different laboratories where there is likely to be substantial variability in staining protocols and measurements. All analyses were performed by Derek A. Hamilton, who was blind to group assignments
} 

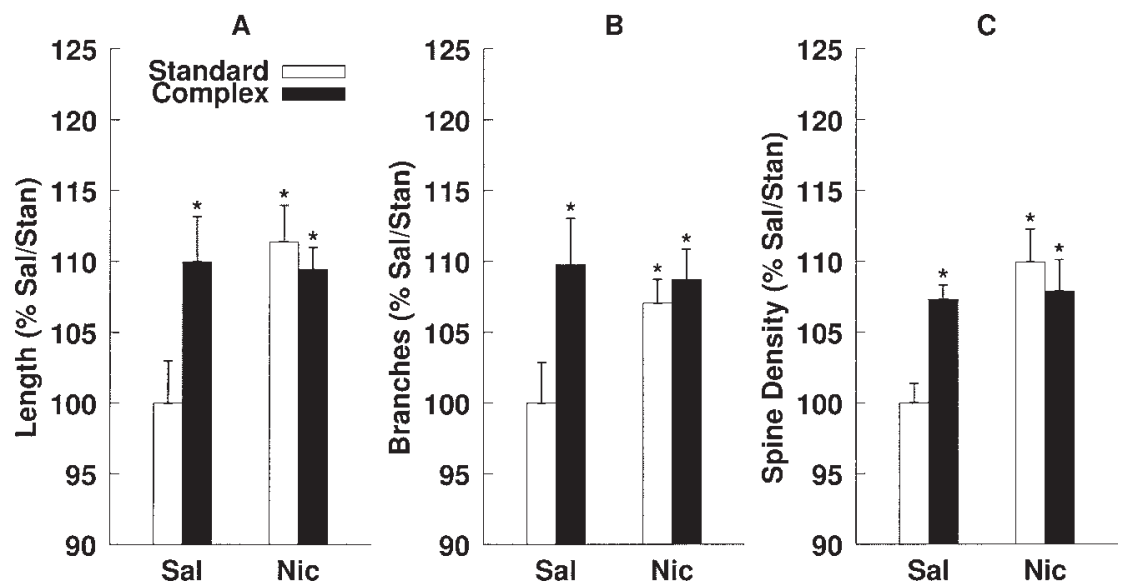

Figure 3. Mean (+1 SEM) dendritic length (A), total branches (B), and spine density (C) in medium spiny neurons of the nucleus accumbens shell for each drug and housing condition in Experiment 1. All values are expressed as the percentage of the saline-treated, standard-housed group (Sal/Stan; $n=5)$. The number of rats in the treatment groups were as follows: saline treated, complex housed $(n=4)$; nicotine treated $(\mathrm{Nic})$, standard housed $(n=5)$; and nicotine treated, complex housed $(n=4)$. The asterisk indicates a significant difference from the Sal/Stan condition at $p<.05$. There were no significant differences between the three treatment conditions for any measure (all $p \mathrm{~s}>.33$ ).

\section{Golgi-Cox Analysis: Par1}

Group means for each measure obtained in Par1 are presented in Table 2. Because no interactions involving hemisphere or hemisphere main effects were statistically significant, the group means were averaged across hemisphere.

Dendritic length. Although complex housing was associated with an increase in apical dendritic length for rats given complex housing regardless of nicotine exposure, the housing main effect was not significant, $F(1,28)=2.87, p=.10$. The drug and hemisphere main effects for apical dendritic length were not significant (both $p s>.43$ ), and none of the two- or three-way interactions for apical dendritic length were significant (all $p \mathrm{~s}>$ .35). Post hoc group comparisons failed to detect significant differences (all $p \mathrm{~s}>.15$ ). For the basilar dendritic field, there was a significant main effect of housing, complex $>$ standard, $F(1$, $28)=6.88, p=.014$, and a main effect of drug, saline $>$ nicotine, $F(1,28)=6.23, p=.019$. None of the two- or three-way interactions reached significance (all $p \mathrm{~s}>.22$ ). Post hoc analyses revealed that rats given complex housing after saline had significantly longer basilar dendrites than saline-treated rats given standard housing ( $p=.012$ ), whereas there was not a significant difference between housing conditions in rats given nicotine ( $p=$ .38).

Dendritic branches. There were no significant effects of drug or housing on apical dendritic branches (both $p$ s $>.18$ ). The hemisphere main effect as well as all of the interaction terms failed to reach significance for apical branches (all $p \mathrm{~s}>.25$ ).

For the basilar dendritic field, there was a significant main effect of housing, complex $>$ standard, $F(1,28)=6.51, p=.016$, and a main effect of drug, saline $>$ nicotine, $F(1,28)=6.51, p=.016$. The Drug $\times$ Housing interaction failed to reach statistical significance, $F(1,28)=3.06$, all $p \mathrm{~s}=.09$, however, inspection of the means in Table 2 suggests that complex housing increased dendritic branching after saline but not nicotine. Post hoc group

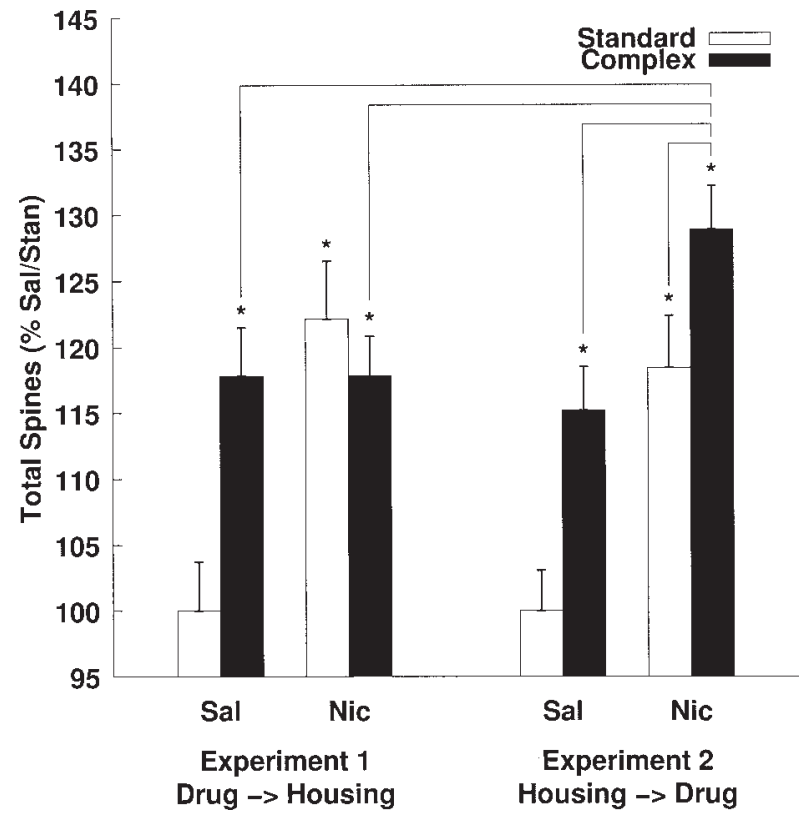

Figure 4. Mean $(+1 S E M)$ total spines for each drug and housing condition from Experiments 1 and 2. Total spine estimates were determined by calculating the product of the dendritic length and spine density measures. All values are expressed as the percentage of the saline-treated, standard-housed group (Sal/Stan) from the same experiment. The number of rats in each group for Experiment 1 were as follows: saline treated, standard housed $(n=5)$; saline treated, complex housed $(n=4)$; nicotine treated (Nic), standard housed $(n=5)$; and nicotine treated, complex housed $(n=4)$. There were 6 rats in each group from Experiment 2. The asterisk indicates a significant difference at $p<.05$ from the Sal/Stan condition from the respective experiment. Lines connect significantly different treatment group means at $p<.05$. 
Table 2

Mean (SEM) Values for Dendritic Length, Branches, Spine Density, and Total Spines in the Apical and Basilar Dendritic Fields of Layer III Pyramidal Neurons in the Parietal Neocortex (Zilles's Par1)

\begin{tabular}{|c|c|c|c|c|}
\hline \multirow[b]{2}{*}{ Dendritic field } & \multicolumn{2}{|c|}{ Saline } & \multicolumn{2}{|c|}{ Nicotine } \\
\hline & Standard & Complex & Standard & Complex \\
\hline \multicolumn{5}{|l|}{ Apical } \\
\hline Length & $100.00(2.84)$ & $108.79(4.54)$ & $100.61(4.63)$ & $106.68(5.83)$ \\
\hline Branches & $100.00(3.88)$ & $107.10(3.93)$ & $97.72(5.29)$ & $97.74(3.63)$ \\
\hline Spine density & $100.00(3.11)_{\mathrm{a}, \mathrm{b}}$ & $113.96(2.72)_{\mathrm{a}, \mathrm{c}}$ & $102.56(3.71)_{\mathrm{c}, \mathrm{d}}$ & $116.17(4.85)_{\mathrm{b}, \mathrm{d}}$ \\
\hline Total spines & $100.00(4.50)_{\mathrm{a}, \mathrm{b}}$ & $123.48(5.19)_{\mathrm{a}, \mathrm{c}}$ & $102.63(4.80)_{\mathrm{c}, \mathrm{d}}$ & $124.46(10.44)_{\mathrm{b}, \mathrm{d}}$ \\
\hline \multicolumn{5}{|l|}{ Basilar } \\
\hline Length & $100.00(3.62)_{a}$ & $112.19(2.91)_{\mathrm{a}, \mathrm{b}, \mathrm{c}}$ & $95.47(3.90)_{\mathrm{b}}$ & $99.59(2.99)_{\mathrm{c}}$ \\
\hline Branches & $100.00(3.09)_{\mathrm{a}}$ & $114.51(4.39)_{\mathrm{a}, \mathrm{b}, \mathrm{c}}$ & $97.29(2.99)_{\mathrm{b}}$ & $100.00(2.50)_{\mathrm{c}}$ \\
\hline Spine density & $100.00(3.31)_{\mathrm{a}}$ & $111.70(4.47)_{\mathrm{a}, \mathrm{b}}$ & $94.19(4.17)_{\mathrm{b}, \mathrm{c}}$ & $106.96(2.84)_{\mathrm{c}}$ \\
\hline Total spines & $100.00(4.21)_{\mathrm{a}}$ & $125.88(6.70)_{\mathrm{a}, \mathrm{b}, \mathrm{c}}$ & $89.90(4.72)_{\mathrm{b}, \mathrm{d}}$ & $106.63(3.78)_{\mathrm{c}, \mathrm{d}}$ \\
\hline
\end{tabular}

Note. All values are expressed as the percentage of the saline-treated, standard-housed rats $(n=5)$. The number of rats in each treatment group for Experiment 1 were as follows: saline treated, complex housed $(n=4)$; nicotine treated, standard housed $(n=5)$; nicotine treated, complex housed $(n=$ 4). Values for groups that share a common subscript for each measure (i.e., in each row) were significantly different at $p<.05$.

comparisons revealed a significant complex-housing-related increase in branches in saline-treated rats $(p=.004)$, an effect that was not observed in rats given nicotine $(p>.56)$. None of the remaining two- or three-way interactions (i.e., those involving hemisphere) or the hemisphere main effect reached significance (all $p \mathrm{~s}>.26$ ).

Spine density. There was a significant main effect of housing on apical spine density, complex $>$ standard, $F(1,28)=12.83$, $p=.001$, whereas the main effect of drug was not significant, $F(1$, 28) $<1, p=.54$. Post hoc comparisons further confirmed that apical spine density in rats given complex housing was significantly higher for saline-treated $(p=.011)$ and nicotine-treated rats $(p=.014)$. None of the interaction terms were significant (all $p$ s $>.59)$. An identical pattern of effects was observed for basilar spine density. There was a significant main effect of housing, complex $>$ standard, $F(1,28)=9.27, p=.005$, whereas the main effect of drug was not significant, $F(1,28)=1.71, p=.20$. Post hoc comparisons confirmed that basilar spine density in rats given complex housing was significantly higher for saline-treated ( $p=$ $.037)$ and nicotine-treated rats $(p=.024)$, suggesting that nicotine did not block the subsequent effects of complex housing on changes in spine density. None of the interaction terms or the hemisphere main effect were significant (all $p \mathrm{~s}>.62$ ).

Total spines. There was a significant housing effect on total apical spines, complex $>$ standard, $F(1,28)=11.43, p=.002$, whereas the main effect of drug was not significant, $F(1,28)<1$, $p=.79$. Post hoc comparisons confirmed that total apical spines in rats given complex housing was significantly higher relative to standard housing for saline-treated $(p=.041)$ and nicotine-treated rats $(p=.021)$. None of the interaction terms for total apical spines were significant (all $p \mathrm{~s}>.46$ ).

There was a significant effect of housing on total basilar spines, complex $>$ standard, $F(1,28)=18.35, p<.001$, as well as a main effect of drug, saline $>$ nicotine, $F(1,28)=8.71, p=.006$. The Drug $\times$ Housing interaction was not significant, $F(1,28)<1, p=$ .37. Post hoc comparisons further confirmed that total basilar spines in rats given complex housing was significantly higher relative to standard housing for saline-treated $(p=.001)$ and nicotine-treated rats $(p=.022)$, thus, despite the overall decrease in total spines due to nicotine, there was no evidence that nicotine blocked total spine increases due to complex housing. None of the remaining interaction terms were significant (all $p \mathrm{~s}>.13$ ).

\section{Discussion}

Consistent with previous reports, complex housing and a dose of nicotine capable of producing hyperactivity produced increases in dendritic length, branching, spine density, and total spine estimates on medium spiny neurons in the NAcc, whereas dendritic changes in pyramidal neurons in Par1 were limited to the complex housing condition. Prior exposure to nicotine limited subsequent structural plasticity in the NAcc (all dendritic measures) and to a lesser degree in Par1 (length and branching only). This pattern of results is similar to the findings recently reported by Kolb, Gorny, Li, et al. (2003) with two other highly addictive psychomotor stimulants (i.e., amphetamine and cocaine). These effects may reflect a competition between forms of experience (drugs and housing) for limited resources necessary for altering dendrites and spines. If so, the ability of psychomotor stimulants to elicit structural changes in the NAcc may also be limited by prior structural changes elicited by complex housing. Given that nicotine produced more higher order branches ( $>5$ th) than complex housing, complex housing may not entirely block structural changes due to nicotine. Experiment 2 was undertaken to investigate these possibilities.

\section{Experiment 2}

To determine whether prior experience influences dendritic changes caused by nicotine, we gave rats complex or standard housing for 2.5 months and subsequently gave them daily injections of nicotine (or saline) for 2 weeks. Dendritic length, branching, spine density, and total spines were quantified on medium spiny neurons in the NAcc shell. Because nicotine alone was not 
found to cause dendritic changes in Par1, this region was not analyzed in Experiment 2.

\section{Method}

At approximately 90 days of age, rats were assigned to standard $(n=12)$ or complex housing $(n=12)$. After 75 days in standard or complex housing, each housing condition was further subdivided into two drug treatment groups (nicotine or saline) and complex-housed rats were transferred to standard housing cages. Rats were given daily injections of nicotine (or saline) for 14 days. Open-field activity was measured 1 day prior to the first injection and following injections on Days 7 and 14. One day following the final injection, brains were extracted and processed for Golgi-Cox staining and analysis.

\section{Results}

\section{Behavior}

As in Experiment 1, nicotine produced increases in open-field activity over the course of the 14-day injection protocol (data not shown). There was a significant Drug $\times$ Day interaction, $F(2$, $44)=16.84, p<.001$, that was attributable to a significant drug effect on the final injection day, nicotine $>$ saline, $F(1,22)=$ $12.74, p=.002$, which was not present 1 day prior to injections or on Injection Day 7 (both $p s>$.17). A comparison of beam crosses on Injection Day 14 for both groups given nicotine failed to detect an effect of complex housing on nicotine-induced hyperactivity, standard housing mean $=4775.5$ (standard error $=539.00)$, complex housing mean $=4767.5$ (standard error $=569.41), F(1$, 10) $<1 ; p=.99$.

\section{Brain Weight}

Mean brain weights for each group from Experiment 2 are shown in Table 1. Brain weights were greater in complex-housed rats, $F(1,20)=6.31, p=.02$. The drug main effect and the Housing $\times$ Drug interaction were not significant (both $p \mathrm{~s}>.32$ ).

\section{Golgi-Cox Analysis: NAcc}

Dendritic length. As shown in Figure 5A, both nicotine and complex housing were associated with increases in dendritic length on medium spiny neurons. There were significant main effects of housing, complex $>$ standard, $F(1,28)=6.49, p=.015$, and drug, nicotine $>$ saline, $F(1,28)=8.83, p=.005$. The Drug $\times$ Housing interaction was not significant, $F(1,28)<1, p=$ .39. Post hoc group comparisons confirmed an effect of nicotine in standard-housed rats $(p=.01)$. Although the change in dendritic length in rats given nicotine after complex housing was approximately $60 \%$ higher compared with rats given saline after complex housing, this difference did not reach post hoc significance ( $p=$ .15). In fact, there were no significant differences among treatment groups (all $p \mathrm{~s}>.15$ ). None of the other interactions with hemisphere or the hemisphere main effect were significant (all $p \mathrm{~s}>$ $.15)$.

Dendritic branches. Figure 5B shows that nicotine and complex housing were associated with increased dendritic branching on medium spiny neurons. There was a significant main effect of drug, nicotine $>$ saline, $F(1,28)=9.63, p=.003$, however, the main effect of housing did not reach significance, $F(1,28)=3.19$, $p=.08$. The Drug $\times$ Housing interaction was not significant, $F(1$, 28) $<1, p=.99$. Post hoc group comparisons confirmed a nicotine-related increase in dendritic branching relative to saline for the standard and complex housing conditions $(p=.029)$. Additional post hoc analyses revealed that both nicotine-treated groups had significantly more higher order (sixth or higher) branches than their respective saline-treated condition (data not shown; both $p \mathrm{~s}<.028$ ), and only rats given nicotine following complex housing had significantly more fifth order branches than baseline (data not shown; $p=.017$ ). None of the interactions with hemisphere or the hemisphere main effect were significant (all $p \mathrm{~s}$ $>$.15).

Spine density. Group means for the spine density measure are shown in Figure 5C. There were significant main effects of hous-
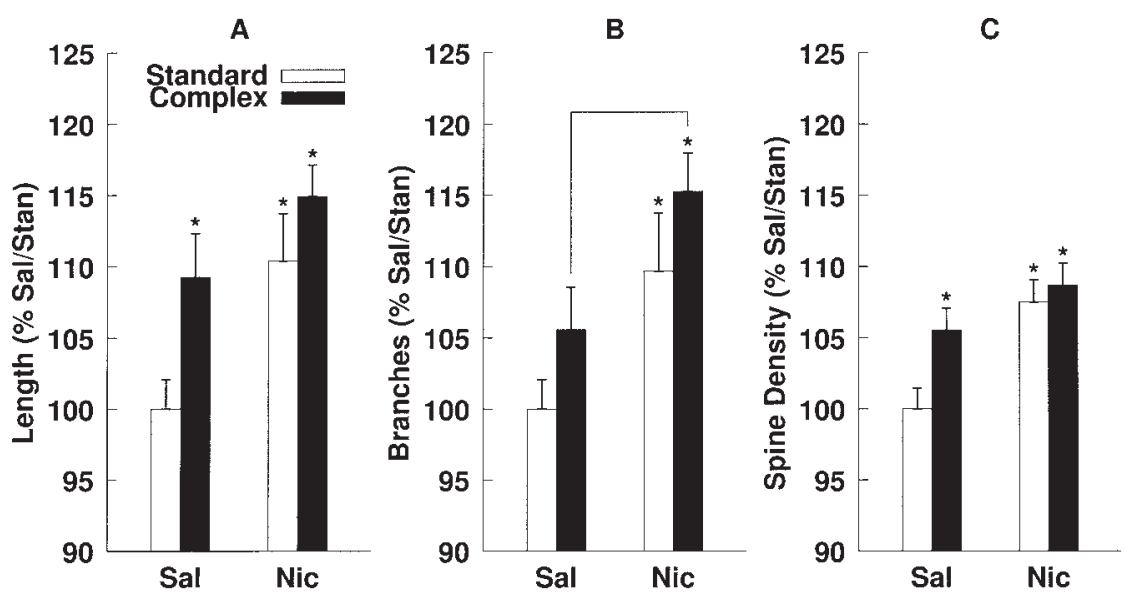

Figure 5. Mean (+1 SEM) dendritic length (A), total branches (B), and spine density (C) in medium spiny neurons of the nucleus accumbens shell for each group $(n=6)$ from Experiment 2. All means are expressed as the percentage of the saline-treated, standard-housed group (Sal/Stan). The asterisk indicates a significant difference from the Sal/Stan condition at $p<.05$. Lines connect significantly different treatment group means at $p<.05$. All other group differences had associated $p s>.15$. Nic $=$ nicotine-treated group. 
ing, complex $>$ standard, $F(1,28)=4.53, p=.04$, and drug, nicotine $>$ saline, $F(1,28)=11.60, p=.002$, and the Drug $\times$ Housing interaction was not significant, $F(1,28)=1.94, p=.17$. Post hoc group comparisons confirmed nicotine-related increases in spine density relative to saline following standard housing ( $p=$ .001 ), however, although nicotine following complex housing led to a $56 \%$ greater increase in spine density relative to saline, this comparison was not significant $(p=.15$ ). None of the interactions involving hemisphere or the hemisphere main effect were significant (all $p \mathrm{~s}>.17$ ).

Total spines. Total spine estimates for each group from Experiment 2 are shown in the right side of Figure 4. There were significant main effects of housing, complex $>$ standard, $F(1$, $28)=10.70, p=.002$, and drug, nicotine $>$ saline, $F(1,28)=$ 17.38, $p<.001$. The Drug $\times$ Housing interaction was not significant, $F(1,28)<1, p=.35$. Post hoc group comparisons confirmed nicotine-related increases in total spines (relative to saline) following standard housing $(p<.001)$ and complex housing $(p=$ $.03)$. All three treatment conditions led to significantly greater total spine estimates relative to the baseline condition (all $p \mathrm{~s}<.003$ ). None of the interactions involving hemisphere or the hemisphere main effect were significant (all $p s>.27$ ).

In Experiment 1, increases in total spines were observed either after nicotine or complex housing, however, nicotine blocked the subsequent increases in spines due to complex housing. In contrast, the results of Experiment 2 demonstrate that complex housing does not block subsequent increases in dendritic spines due to nicotine. The critical comparison between rats given nicotine and complex housing in both experiments confirmed that significantly higher total spine estimates were obtained when rats were given nicotine after complex housing relative to rats given nicotine before complex housing ( $p=.037$ ). This rules out a ceiling effect as an explanation for why additive effects of nicotine and complex housing were not observed in Experiment 1. Additional post hoc group comparisons among the 8 groups from Experiments 1 and 2 (see Figure 4) confirmed that rats given nicotine following complex housing in Experiment 2 had significantly higher total spine estimates than all other conditions from both experiments (all $p \mathrm{~s}<$ .037), with the exception of rats given nicotine then standard housing in Experiment $1(p=.17)$.

\section{Discussion}

In contrast to the effects of nicotine on subsequent structural plasticity observed in Experiment 1, prior experience with complex housing did not block structural plasticity due to nicotine. Nicotine given after complex housing was associated with more dendritic branching and total spines compared with rats given saline following complex housing or standard housing. The relative effects of nicotine and complex housing on subsequent structural plasticity was particularly evident in the effects on total spines. Whereas exposure to complex housing after nicotine (in Experiment 1) was associated with a negligible change in total spines relative to rats that only received nicotine, exposure to nicotine after complex housing led to increases in spines above those obtained with either nicotine or complex housing alone. Rats given nicotine after complex housing also had more dendritic branches than rats given saline after complex housing. This effect was likely due to the fact that nicotine produced increases in higher order branches after both standard and complex housing, increases that were not observed due to complex housing alone. Complex housing causes widespread changes in the brain, as evidenced by the effects on Par1 in Experiment 1 and the increases in gross brain weight. These changes, however, were not sufficient to block the subsequent morphological effects of nicotine. That the effects of nicotine on motor hyperactivity were also unaffected by prior complex housing provides evidence that complex housing did not substantially impact the subsequent behavioral effects of nicotine. In contrast to the effects of Experiment 2, additive effects of nicotine and complex housing on dendritic branching were not observed in Experiment 1. It is important to note that the additive effects of nicotine and complex housing on spines and branching in Experiment 2 rule out a ceiling effect as an explanation for why complex housing did not elicit structural changes in NAcc after nicotine in Experiment 1.

\section{General Discussion}

The major results of the present study support the conclusion that (a) nicotine blocks subsequent effects of complex housing structural plasticity in NAcc and Par1 and that (b) complex housing does not block subsequent effects of nicotine structural plasticity in NAcc. The measures we used (dendritic length, branching, spine density, and total spines) have previously been used to demonstrate the effects of complex housing (Kolb, Gorny, Soderpalm, \& Robinson, 2003) and stimulant drugs on dendritic structure in the investigated brain regions (Brown \& Kolb, 2001; Kolb, Gorny, Li, et al., 2003; Robinson \& Kolb, 1999). These measures provide indirect information about synaptic organization, and experience-dependent changes in these measures are considered to reflect a change in the amount of space available for synaptic connections. In Experiment 1, changes in dendritic structure in NAcc (all measures) and Par1 (length and branching only) due to complex housing were observed following treatment with saline but not nicotine. These results suggest that, like amphetamine and cocaine (Kolb, Gorny, Li, et al., 2003), prior nicotine exposure diminishes the ability of individual neurons in NAcc and Par1 to undergo subsequent plastic change(s) related to experience. In contrast, the results of Experiment 2 demonstrate that nicotine can elicit changes in dendritic structure following prior experience with complex housing. Total spine and dendritic branching increases in NAcc due to nicotine were not affected by prior complex housing. An important implication of these results is that stimulant drugs may simply be more effective in overcoming limits on structural plasticity imposed by prior experience.

Because the cellular and molecular effects of stimulant drugs and experience appear to be similar, it has been suggested that drugs hijack the normal mechanisms involved in synaptic plasticity. Therefore, exposure to stimulant drugs may interfere with plasticity due to other forms of experience. The results of Experiment 1 in Parl and NAcc support this hypothesis. One possibility is that the resources available for the type of structural change investigated here are limited, placing an upper bound on the amount of change that can occur. The data from Experiment 1 cannot rule out a ceiling effect, as similar changes in dendritic structure were observed in all three groups receiving complex housing, nicotine, or both, relative to baseline. The results of Experiment 2 indicate that prior experience (complex housing) did 
not block structural changes in NAcc dendrites due to nicotine. Rats given nicotine after complex housing had significantly higher total spine estimates and branches than rats given saline after complex housing. In both experiments, nicotine treatment was associated with significant increases in higher order branches $(>$ 5 th) relative to complex housing alone, thus, nicotine had unique effects on branches that were preserved following complex housing. The differential effects of prior nicotine treatment or complex housing on subsequent structural plasticity are evidenced by the fact that nicotine after complex housing was associated with a significantly greater increase in total spines compared with rats given complex housing after nicotine (Experiment 1). This result also effectively rules out the possibility of a ceiling effect in Experiment 1. Thus, the competition among different forms of experience with respect to structural plasticity appears to be more complicated and will require additional research to explain.

Because both complex housing and nicotine exposure increase the expression of basic fibroblast growth factor in several brain regions, including NAcc (Belluardo, Blum, Mudo, Andbjer, \& Fuxe, 1998; Kolb et al., 1998), it is possible that the differential effects of housing and nicotine on subsequent structural plasticity may be related to differential effects on the expression of basic fibroblast growth factor or other neurotrophic factors. We are unaware of any studies that have directly compared the presence of neurotrophic factors in NAcc after nicotine exposure or complex housing. On the basis of the results obtained here, one might expect that the levels of growth factor produced by nicotine may exceed those due to experience. Of course, a difference in the expression of neurotrophic factors and in synaptic plasticity may be due to effects on cellular events that precede expression of these changes, perhaps as early as activity-regulated gene expression. It is interesting that stimulants and complex experience have comparable effects on immediate early gene (IEG) expression. For example, amphetamine (Klebaur et al., 2002; Kodama et al., 1998) and environmental enrichment (Pinaud, Penner, Robertson, \& Currie, 2001) both modify the expression of the IEG Arc in NAcc, which has been linked to learning, synaptic plasticity, and neural activation in other brain regions (Guzowski, McNaughton, Barnes, \& Worley, 1999; Steward, Wallace, Lyford, \& Worley, 1998; Vazdarjanova, McNaughton, Barnes, Worley, \& Guzowski, 2002). Although there are currently no available data regarding the effects of nicotine on IEG Arc expression in NAcc, it might be expected that nicotine will be associated with larger effects than experience. Of course, differences in experience-dependent structural plasticity may also be related to other stages in the cascade of events intervening between gene expression and protein production (e.g., transcription and posttranscription regulation).

As mentioned, the asymmetry in the effects of nicotine and complex housing on subsequent structural plasticity may reflect a fundamental difference in the effectiveness of nicotine and normal experience to elicit structural changes in neurons. Even the relatively low dose of nicotine we used over the 2-week injection period consistently caused larger numerical changes in NAcc dendrites than 2.5-3.0 months of complex housing. In contrast, consistently higher gross brain weights were observed in rats given complex housing, whereas evidence for increases in brain weight after nicotine were weak and only present 3 months after cessation (Experiment 1). Complex housing also produces dendritic changes throughout the neocortical mantle, which are likely due to the diverse types of experience that are available to the rat. Less is known regarding the regions where nicotine produces structural changes, although they are likely to be fewer in number than those altered by complex housing. Thus, perhaps the observed asymmetry is related to differences in the number of brain regions that undergo dendritic changes, with nicotine exerting a more focused effect on fewer regions. Future studies should replicate the experiments reported here using types of experience that affect a more limited number of brain regions and that have some overlap with the regions affected by nicotine.

Nicotine and complex housing both produced increases in dendritic measurements, thus, it could be argued that effects of nicotine and complex housing arise because these types of experience simply provide some form of nonspecific stimulation compared with the relatively unstimulating standard housing condition. Such an explanation cannot be ruled out on the basis of the results of Experiment 1 alone, because nicotine, complex housing, or both together were associated with comparable differences from the standard housing condition. The results of Experiment 2 demonstrate that providing complex housing prior to nicotine causes greater changes in dendritic structure than when complex housing is given after nicotine (in Experiment 1). If experience-dependent increases in dendritic morphology simply reflect a simplification of dendritic fields in control brains or nonspecific effects of stimulation, then the dendritic changes following both nicotine and complex housing should not depend on order. The idea that apparent experience-dependent changes in dendritic structure are related to simplification in comparison brains or nonspecific increases correlated with experience also cannot explain why complex housing and nicotine are associated with decreases in dendritic arbor and spines in some brain regions relative to standardhoused rats (Hamilton, Silasi, Carroll, Pellis, \& Kolb, 2004; Kolb, Gibb, \& Gorny, 2003). A more parsimonious explanation is that dendritic changes (increases or decreases) occur in response to experience and are specific to dendrites and spines within the neural circuitry engaged by the experience.

Because dendritic spines are the primary sites of excitatory synapses and are thought to be the primary locus where plastic changes affect synaptic signaling (Harris \& Kater, 1994; Nimchinsky et al., 2002), increases in spines are likely to have an important impact on how individual neurons process afferent signals and ultimately affect activity within and between neural networks. The results of the present study suggest that prior nicotine and complex housing should have different effects on neural activity, which may lead to important differences in the long-term consequences for subsequent cognition and behavioral adaptation (i.e., learning). Indeed, a small but growing body of literature indicates that stimulant addicts experience a diverse array of neuropsychological impairments including problems with learning and memory (Bolla, Cadet, \& London, 1998; Rogers \& Robbins, 2001). Of course, a more convincing demonstration of this significance as it relates to the present data would involve demonstrating a clear effect of prior nicotine exposure on subsequent learning. Gonzalez, Kolb, and colleagues (Gonzalez, 2004; Kolb, Gonzalez, Gharbawie, Whishaw, \& Hamilton, 2004) have found that giving the dose of nicotine used in the present studies interfered with subsequent learning in a skilled reaching task known to cause increased dendritic branching and length in Layer V forelimb area neurons (Kolb, 1995). The same dose of nicotine was also found to cause 
increased dendritic length and branching in Layer $\mathrm{V}$ forelimb area neurons (Kolb et al., 2004). Thus, nicotine-induced dendritic enhancement is associated with impaired learning that normally causes dendritic enhancement in the same population of neurons.

Although the effects of prior nicotine or complex housing on subsequent structural plasticity represent the major novel findings of the present study, this is also the first demonstration, to our knowledge, that the effects of nicotine on dendritic structure in NAcc persist for at least 3 months after cessation. The effects of nicotine on NAcc dendritic structure were comparable whether the rats were euthanized immediately (Experiment 2) or 90 days (Experiment 1) after cessation. This raises questions regarding the duration of nicotine-induced alterations in dendritic morphology and the potential extent to which the behavioral and cognitive consequences of nicotine exposure persist. Future experiments should be undertaken to systematically evaluate the duration of these effects. Because the effects of amphetamine and nicotine on behavior and dendritic morphology appear to be similar, one might expect the duration of nicotine-induced changes in behavior and NAcc to last up to a year in the rat, given that behavioral sensitization to amphetamine lasts at least that long (Paulson et al., 1991).

One important difference between the outcomes observed here and those from previous studies concerns the degree of change induced by nicotine. The changes in dendritic structure due to nicotine in the present experiment were substantially lower than those reported by Brown and Kolb (2001), but were comparable with those observed by Kolb, Gorny, Li, et al. (2003) with amphetamine and cocaine. We used a substantially lower dose of nicotine $(0.3 \mathrm{mg} / \mathrm{kg}$ vs. $0.7 \mathrm{mg} / \mathrm{kg})$, a different type of nicotine (salt vs. free base liquid), and fewer total injections (14 vs. 36) than Brown and Kolb (2001). Brown and Kolb demonstrated nicotineinduced changes in dendritic length on NAcc neurons on the order of 30\%-40\% relative to control animals, whereas the dose we used led to increases on the order of $10 \%$. It is important to note that the relatively small dose of nicotine we used was still sufficient to cause long-lasting changes in dendritic morphology in the NAcc. Nonetheless, the ability of nicotine to block subsequent structural plasticity and to induce structural changes in dendrites after complex housing may be related to dose, thus, other studies should be undertaken to systematically investigate dose effects. Given that the injection regimen we used led to behavioral effects that emerged between 7 and 14 days after the first injection, cumulative doses ranging from $2.1-4.2 \mathrm{mg} / \mathrm{kg}$ may be sufficient to cause the effects reported here.

In summary, the present results indicate that drugs and complex housing differ in their capacity to influence subsequent experience-dependent changes in dendritic structure. The effects of chronic drug use are commonly attributed to neural insults (brain damage), however, the present results and the results of Kolb, Gorny, Li, et al. (2003) suggest that at least some of the psychological effects associated with stimulant use may be related to subsequent limits on structural plasticity rather than frank damage. In the present study, nicotine was found to block subsequent dendritic modifications associated with experience. Kolb, Gorny, $\mathrm{Li}$, et al. (2003) speculated that one positive consequence of prior experience would be a blockade or attenuation of the subsequent effects of stimulant drugs. In contrast to this prediction, nicotine induced additional structural modifications in dendrites and increased motor hyperactivity after complex housing. Thus, the promising possibility that environmental experience in adulthood can exert a protective effect against the subsequent effects of stimulant drugs is not supported. The present results underscore the importance of systematically evaluating the behavioral and neural consequences of stimulant exposure, an undertaking that takes on added importance when one considers widespread abuse of stimulant drugs and the growing range of patient populations in which stimulants are used for therapeutic purposes.

\section{References}

Belluardo, N., Blum, M., Mudo, G., Andbjer, B., \& Fuxe, K. (1998). Acute intermittent nicotine treatment produces regional increases of basic fibroblast growth factor messenger RNA and protein in the tel- and diencephalon of the rat. Neuroscience, 83, 723-740.

Bernert, G., Sustrova, M., Sovcikova, E., Seidl, R., \& Lubec, G. (2001). Effects of a single transdermal nicotine dose on cognitive performance in adults with Down syndrome. Journal of Neural Transmission 61(Suppl.), 237-245.

Berridge, K. C., \& Robinson, T. E. (2003). Parsing reward. Trends in Neurosciences, 26, 507-513.

Bolla, K. I., Cadet, J. L., \& London, E. D. (1998). The neuropsychiatry of chronic cocaine abuse. Journal of Neuropsychiatry and Clinical Neurosciences, 10, 280-289.

Brown, R. W., \& Kolb, B. (2001). Nicotine sensitization increases dendritic length and spine density in the nucleus accumbens and cingulate cortex. Brain Research, 899, 94-100.

Coleman, P. D., \& Riesen, A. H. (1968). Environmental effects on cortical dendritic fields: I. Rearing in the dark. Journal of Anatomy, 102, 363374.

Collins, A. C., Romm, E., \& Wehner, J. M. (1990). Dissociation of the apparent relationship between nicotine tolerance and up-regulation of nicotinic receptors. Brain Research Bulletin, 25, 373-379.

Fiala, J. C., Spacek, J., \& Harris, K. M. (2002). Dendritic spine pathology: Cause or consequence of neurological disorders. Brain Research Reviews, 39, 29-54.

Fung, Y. K., \& Lau, Y. S. (1989). Effects of prenatal nicotine exposure on rat striatal dopaminergic and nicotinic systems. Pharmacology Biochemistry and Behavior, 33, 1-6.

Gibb, R., \& Kolb, B. (1998). A method for Golgi-Cox staining of Vibratome cue tissue. Journal of Neuroscience Methods, 79, 1-4.

Glaser, E. M., \& Van der Loos, H. (1981). Analysis of thick brain sections by obverse-reverse computer microscopy: Application of a new, high clarity Golgi-Nissl stain. Journal of Neuroscience Methods, 4, 117-125.

Gonzalez, C. L. R. (2004). An analysis of poststroke motor dysfunction and cerebral reorganization in the rat. Unpublished doctoral dissertation, University of Lethbridge, Lethbridge, Alberta, Canada.

Greenough, W. T., \& Bailey, C. H. (1988). The anatomy of a memory: Convergence of results across a diversity of tests. Trends in Neurosciences, 11, 142-147.

Greenough, W. T., Withers, G. S., \& Wallace, C. S. (1990). Morphological changes in the nervous system arising from behavioral experience: What is the evidence that they are involved in learning and memory? In L. R. Squire \& E. Lindenlaub (Eds.), The biology of memory: Vol. 23. Symposia medica hoechst (pp. 159-185). New York: Schattauder.

Guzowski, J. F., McNaughton, B. L., Barnes, C. A., \& Worley, P. F. (1999). Environment-specific expression of the immediate-early gene Arc in hippocampal neuronal ensembles. Nature Neuroscience, 2, 1120 1124.

Hamilton, D. A., Silasi, G., Carroll, C., Pellis, S. M., \& Kolb, B. (2004, October). Experience differentially affects the orbital and medial prefrontal cortex of the rat [Abstract]. Poster session presented at the annual meeting of the Society for Neuroscience (Program No. 771.16). Retrieved from http://sfn.scholarone.com. 
Harris, K. M., \& Kater, S. B. (1994). Dendritic spines: Cellular specializations imparting both stability and flexibility to synaptic function. Annual Review of Neuroscience, 17, 341-371.

Jarvik, M. E. (1991). Beneficial effects of nicotine. British Journal of Addiction, 86, 571-575.

Kelley, A. E. (1999). Neural integrative activities of nucleus accumbens subregions in relation to learning and motivation. Psychobiology, 27, $198-213$

Klebaur, J. E., Ostrander, M. M., Norton, C. S., Watson, S. J., Akil, H., \& Robinson, T. E. (2002). The ability of amphetamine to evoke Arc (Arg 3.1) mRNA expression in the caudate, nucleus accumbens and neocortex is modulated by environmental context. Brain Research, 930, 30-36.

Kodama, M., Akiyama, K., Ujike, H., Shimizu, Y., Tanaka, Y., \& Kuroda, S. (1998). A robust increase in expression of Arc gene, an effector immediate early gene, in the rat brain after acute and chronic methamphetamine administration. Brain Research, 796, 273-283.

Kolb, B. (1995). Brain plasticity and behavior. Mahwah, NJ: Erlbaum.

Kolb, B., Forgie, M., Gibb, R., Gorny, G., \& Rowntree, S. (1998). Age, experience and the changing brain. Neuroscience and Biobehavioral Reviews, 22, 143-159.

Kolb, B., Gibb, R., \& Gorny, G. (2003). Experience-dependent changes in dendritic arbor and spine density in neocortex vary qualitatively with age and sex. Neurobiology of Learning and Memory, 79, 1-10.

Kolb, B. E., Gonzalez, C. L., Gharbawie, O., Whishaw, I. Q., \& Hamilton, D. A. (2004). Prior exposure to nicotine blocks experience-dependent changes in dendritic morphology and behavior [Abstract]. Poster session presented at the annual meeting of the Society for Neuroscience (Program No. 120.6). Retrieved from http://sfn.scholarone.com.

Kolb, B., Gorny, G., Li, Y., Samaha, A. N., \& Robinson, T. E. (2003). Amphetamine or cocaine limits the ability of later experience to promote structural plasticity in the neocortex and nucleus accumbens. Proceedings of the National Academy of Sciences, USA, 100, 10523-10528.

Kolb, B., Gorny, G., Soderpalm, A. H. V., \& Robinson, T. E. (2003). Environmental complexity has different effects on the structure of neurons in the prefrontal cortex versus the parietal cortex or nucleus accumbens. Synapse, 48, 149-153.

Kolb, B., \& Whishaw, I. Q. (1998). Brain plasticity and behavior. Annual Review of Psychology, 49, 43-64.

Le Houezec, J., Halliday, R., Benowitz, N. L., Callaway, E., Naylor, H., \& Herzig, K. (1994). A low dose of subcutaneous nicotine improves information processing in non-smokers. Psychopharmacology, 114, $628-634$

Levin, E. D., Wilkerson, A., Jones, J. P., Christopher, N. C., \& Briggs, S. J. (1996). Prenatal nicotine effects on memory in rats: Pharmacological and behavioral challenges. Developmental Brain Research, 97, 207-215.

Li, Y., Kolb, B., \& Robinson, T. E. (2003). The location of persistent amphetamine-induced changes in the density of dendritic spines on medium spiny neurons in the nucleus accumbens and caudate-putamen. Neuropsychopharmacology, 28, 1082-1085.

Miyata, H., \& Yanagita, T. (2001). Neurobiological mechanisms of nicotine craving. Alcohol, 24, 87-93.

Murrin, L. C., Ferrer, J. R., Zeng, W. Y., \& Haley, N. J. (1987). Nicotine administration to rats: Methodological considerations. Life Sciences, 40, $1699-1708$

Nimchinsky, E. A., Sabatini, B. L., \& Svoboda, K. (2002). Structure and function of dendritic spines. Annual Review of Physiology, 64, 313-353.

Paulson, P. E., Camp, D. M., \& Robinson, T. E. (1991). Time course of transient behavioral depression and persistent behavioral sensitization in relation to regional brain monoamine concentrations during amphetamine withdrawal in rats. Psychopharmacology, 103, 480-492.

Pich, E. M., Pagliusi, S. R., Tessari, M., Talabot-Ayer, D., van Huijsduijnen, R. H. \& Chiamulera, C. (1997, January 3). Common neural substrates for the addictive properties of nicotine and cocaine. Science, $275,83-86$.

Pinaud, R., Penner, M. R., Robertson, H. A., \& Currie, R. W. (2001). Upregulation of the immediate early gene $A r c$ in the brains of rats exposed to environmental enrichment: Implications for molecular plasticity. Molecular Brain Research, 91, 50-56.

Purpura, D. P. (1974, December 20). Dendritic spine dysgenesis and mental retardation. Science, 186, 1126-1128.

Rezvani, A. H., \& Levin, E. D. (2001). Cognitive effects of nicotine. Biological Psychiatry, 49, 258-267.

Robinson, T. E., \& Kolb, B. (1997). Persistent structural modifications in nucleus accumbens and prefrontal cortex neurons produced by previous experience with amphetamine. Journal of Neuroscience, 17, 8491-8497.

Robinson, T. E., \& Kolb, B. (1999). Alterations in the morphology of dendrites and dendritic spines in the nucleus accumbens and prefrontal cortex following repeated treatment with amphetamine or cocaine. European Journal of Neuroscience, 11, 1598-1604.

Rogers, R. D., \& Robbins, T. W. (2001). Investigating the neurocognitive deficits associated with chronic drug misuse. Current Opinion in Neurobiology, 11, 250-257.

Rosenzweig, M. R., \& Bennett, E. L. (1978). Experiential influences on brain anatomy and brain chemistry in rodents. In G. Gottlieb (Ed.), Studies on the development of behavior and the nervous system (pp. 289-387). New York: Academic Press.

Rosenzweig, M. R., Krech, D., Bennett, E. L., \& Diamond, M. C. (1962). Effects of environmental complexity and training on brain chemistry and anatomy: A replication and extension. Journal of Comparative and Physiological Psychology, 55, 429-437.

Sholl, D. A. (1981). The organization of the cerebral cortex. London: Methuen.

Steward, O., Wallace, C. S., Lyford, G. L., \& Worley, P. F. (1998). Synaptic activation causes the mRNA for the IEG $\operatorname{Arc}$ to localize selectively near activated postsynaptic sites on dendrites. Neuron, 21, $741-751$.

Trauth, J. A., Seidler, F. J., McCook, E. C., \& Slotkin, T. A. (1999). Adolescent nicotine exposure causes persistent upregulation of nicotinic cholinergic receptors in rat brain regions. Brain Research, 851, 9-19.

van Pragg, H., Kempermann, G., \& Gage, F. (2000). Neural consequences of environmental enrichment. Nature Reviews Neuroscience, 1, 191198.

Vazdarjanova, A., McNaughton, B. L., Barnes, C. A., Worley, P. F., \& Guzowski, J. F. (2002). Experience-dependent coincident expression of the effector immediate-early genes Arc and Homer-1a in hippocampal and neocortical neuronal networks. Journal of Neuroscience, 22, 1006710071.

Zilles, K. (1985). The cerebral cortex of the rat. Berlin, Germany: Springer-Verlag.

Received April 30, 2004

Revision received September 7, 2004

Accepted October 15, 2004 